\title{
Habit and morphology study on the palm-based 9,10-dihydroxystearic acid (DHSA) crystals
}

\begin{abstract}
Dihydroxystearic acid (DHSA) and its derivatives are hydroxyl fatty acids suitable to be used as multipurpose intermediates in the synthesis of personal care products and decorative cosmetics. In Malaysia, DHSA has been successfully produced from palm-based oleic acid, via epoxidation with per-formic acid followed with hydrolysis of the epoxide. The objective of this paper is to study the crystals of DHS A produced under different crystallization conditions. The crystal habit and morphology were observed in terms of scanning electron microscopy (SEM) and X-ray diffraction (XRD). Results show that solvent type, solvent concentration and cooling mode affect the crystal habit but not the morphology. The DHSA crystals agglomerated into either sphere-like or plate-like habit structure while always maintaining the triclinic crystal system.
\end{abstract}

Keyword: Crystal structure; Crystallization; Electron microscopy; X-ray diffraction topography 